\title{
IMPORTÃNCIA DOS ANELÍDEOS POLIQUETAS NA ALIMENTAÇÃO DA MACROFAUNA DEMERSAL E EPIBENTÔNICA DA REGIÃO DE UBATUBA
}

\author{
A. CECILIA Z. AMARAL* e ALVARO E. MIGOTTO \\ Instituto Oceanografico, Universidade de Sø̃o Paulo, Sø̊o Paulo, SP, Brasil
}

\section{SYNOPSIS}

The purpose of the present investigation is to evaluate the importance of the contribution of polychaetous annelids to the feeding habits of fishes, crustaceans and molluscs from an important fishing area to the northern coast of São Paulo. Many of the species of fishes caught by otter-trawl, along the first phase of the present work have shown a remarkable preference for the polychaetes, as food items. Among them Rhinobatos horkelli, Orthopristis ruber. Cynoscion striatus, Menticirrhus americanus, Micropogon furnieri, Paralonchurus brasiliensis, Umbrina canosai, Etropus intermedius and Symphurus trewavasae are the most representative in this respect. The taxonomic analysis of the worms revealed the presence of 32 species, from which the more frequent was Nothria stigmatis, Pherusa laevis, Pherusa parmata, Piromis arenosus, Pectinaria laelia and Thelepus setosus.

\section{Introdução}

O conhecimento do regime alimentar é de extrema importáncia para a compreensão de muitos processos biológicos e para a exploração racional de estoques de peixes e de outros organismos marinhos de valor econômico.

Os poliquetos contribuem com mais de $80 \%$ do alimento ingerido por algumas espécies de peixes, participando ainda significativamente da cadeia alimentar das populaçōes bentônicas, como já tem sido evidenciado por vários autores. Perkins \& Savage (1975) fizeram uma revisão da bibliografia recente e evidenciaram o interesse e alcance dos estudos efetuados nesse campo.

A preferência de muitos peixes marinhos, pelos poliquetos como alimento, é indiretamente evidenciada pela utilização desses anelídeos como isca. De acordo com 1'aylor \& Saloman (1968), em 1966 foram comercializados nos Estados Unidos, com tal finalidade, 750 toneladas de poliquetos com valor total de US\$ 1.3 milhōes.
O projeto do qual o presente trabalho faz parte propõe-se ao estudo de uma possível correlação entre a abundância de determinadas espécies de poliquetos e a ocorrência de organismos demersais de interesse econômico, ou ainda, coincidência das áreas de distribuição do alimento e dos consumidores.

Nesta fase prelıminar sáo avaliados os resultados obtidos com a análise do conteúdo estomacal de todos os exemplares presentes nas amostras, tendo por objetivo avaliar a importância da participação dos anelídeos poliquetos nos respectivos regimes alimentares.

\section{Material e Métodos}

A área escolhida está próxima ao laboratório costeiro do Instituto Oceanográfico da Universidade de São Paulo, no Município de Ubatuba, Estado de São Paulo, sendo compreendida entre a enseada do Flamengo, Ilha Vitória e enseada da Picinguaba (Fig. 1).

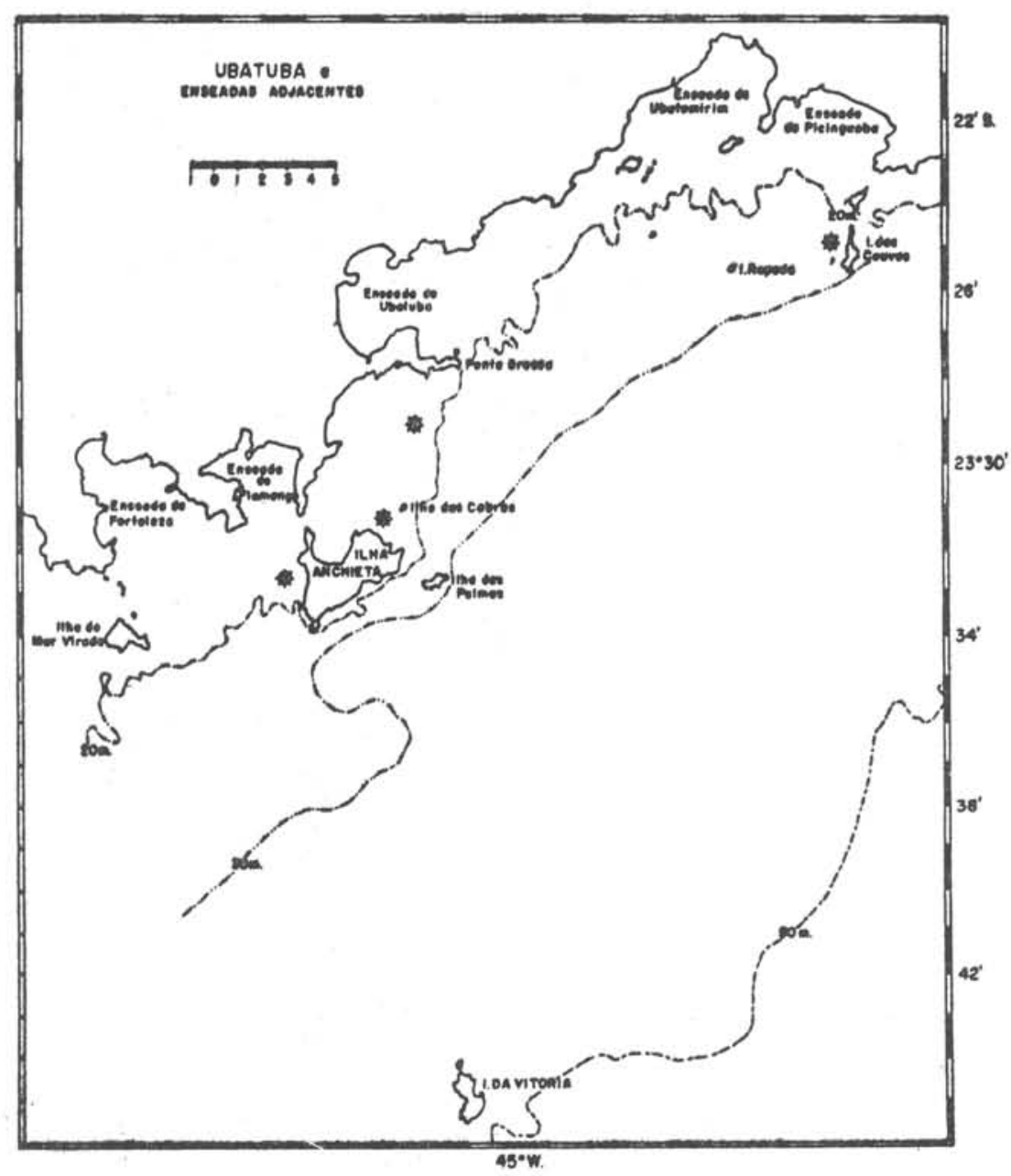

Fig. 1 - Mapa da região, assinalando a área amostrada. 
As coletas foram realizadas utilizando-se redes-de-arrasto-de porta ("otter-trawl") visando a captura da macrofauna demersal e epibentônica, nas profundidas de 10-50 metros. 0 tempo de arrasto foi de 30-60 minutos, conforme observação local por meio de sondagem contínua, com velocidade aproximada de 2,0 nós.

Os exemplares estudados nesta fase foram capturados de março a junho de 1978, ao largo das ilhas Anchieta, das Couves, das Cabras, bem como entre Ponta Grossa de Ubatuba e a ilha das Cabras.

Após uma primeira triagem a bordo, as amostras foram mantidas a baixa temperatura em caixas de isopor com gelo, e o barco regressou imediatamente. visando assegurar uma boa conservação do conteúdo do trato digestivo. Essa precaução é importante porque, em muitos casos, os processos digestivos são extremamente rápidos.
Os tratos digestivos foram conservados em formol a $10 \%$ e posteriormente dissecados e o volume total do conteúdo estomacal, quando mensurável, determinado pelo método do deslocamente, utilizandose provetas graduadas. O conteúdo foi examinado, sendo anotados os organismos presentes. Atenção especial foi dispensada aos poliquetos, procurando-se conhecer a frequência e importância de cada família, gênero ou, quando possível, de cada espécie.

\section{Resultados e Discussão}

Das espécies coletadas, as relacionadas na Tabela $\mathrm{I}$, tiveram seu conteúdo estomacal analisado.

TABELA 1

ESPECIES ESTUDADAS, NUMERO DE ESTOMAGOS EXAMINADOS (A) e NUMERO DE ESTOMAGOS COM POLIQUETOS (B)

\begin{tabular}{|c|c|c|c|c|c|}
\hline Especie & A & B & Espécie & $\mathbf{A}$ & B \\
\hline Octopus sp & 2 & - & Orthopristis ruber & 18 & 16 \\
\hline Achaelus spinimanus & 11 & 2 & Ctenosciaena gracilicirrhus & 1 & - \\
\hline Arenoeus cribrarius & 5 & - & Cynoscion jamaicensis & 3 & 1 \\
\hline Libia sp & 2 & 1 & Cynoscion striatus & 14 & 8 \\
\hline Squatina argentina & 9 & 4 & Cynoscion virescens & 2 & - \\
\hline Rhinobatos horkelli & 9 & 4 & Isopisthus parvipinnis & 23 & - \\
\hline Zapteryx brevirostris & 12 & 7 & Larimus breviceps & 2 & - \\
\hline Raja agassizi & 20 & - & Menticirrhus americanus & 69 & 18 \\
\hline Sympterygia acuta & 4 & - & Micropogon furnieri & 44 & 35 \\
\hline Narcine brasiliensis & 11 & 9 & Ophioscium microps & 2 & 1 \\
\hline Gymnotorax ocellatus & 7 & - & Ophioscium punctatissimus & 2 & 1 \\
\hline Harengula clupeola & 1 & - & Paralonchurus brasiliensis & 112 & 105 \\
\hline Anchoa spinifera & 1 & - & Umbrina canosai & 6 & 6 \\
\hline Synodus foetens & 5 & - & Umbrina coroides & 13 & 3 \\
\hline Trachinochephalus myops & 7 & - & Upeneus parvus & 6 & 2 \\
\hline Genidens genidens & 6 & 2 & Chaetodipterus faber & 5 & 1 \\
\hline Netuma barba & 8 & 4 & Sphyraena picudilla & 11 & 8 \\
\hline Porichthys prosissimus & 5 & - & Thyrsitops lepidopoides & 2 & - \\
\hline Phrinelox scaber & 1 & - & Trichiurus lepturus & 1 & - \\
\hline Ogcocephalus vespertilio & 1 & - & Peprilus paru & 1 & - \\
\hline Prionotus alipionis & 23 & 1 & Citharrichthys cornutus & 10 & - \\
\hline Dactylopterus volitans & 13 & 2 & Cyclopsetta chittendeni & 4 & - \\
\hline Diplectrum formosum & 1 & - & Etropus intermedius & 25 & 23 \\
\hline Diplectrum radiale & 16 & 2 & Paralichthys patagonicus & 4 & - \\
\hline Epinephelus niveatus & 2 & - & Scyacium microrum & 1 & - \\
\hline Priacantus arenatus & 2 & 1 & Scyacium papilossum & 3 & - \\
\hline Chloroscombrus chrysurus & 3 & - & Symphurus diomedianus & 1 & - \\
\hline Selene vomer & 1 & - & Symphurus jenynsil & 15 & 3 \\
\hline Lutjanus sinagris & 4 & 2 & Symphurus plagusia & 2 & - \\
\hline Eucinostomus argenteus & 2 & 2 & Symphurus trewavasae & 7 & 3 \\
\hline Eucinostomus gula & 11 & 5 & Stephanoleppis hispidus & 13 & 6 \\
\hline Eugerres sp & 4 & 2 & Lagocephalus laevigatus & 5 & 1 \\
\hline Conodon nobilis & 46 & 14 & Sphoeroides pachigaster & 1 & - \\
\hline Haemulon striatum & 5 & 2 & Chilomycterus spinosus & 15 & 6 \\
\hline
\end{tabular}

Os anelídeos poliquetos foram o principal componente do conteúdo do trato digestivo das espécies : Squatina argentina, Rhino. batos horkelli, Zapteryx brevinostris, Narcine brasiliensis, Netuma barba, Eucinostomus gula, Conodon nobilis, Orthopristis ruber, Cynoscion striatus, Menticirrhus americanus, Micropogon furnieri Paralonchurus brasiliensis, Umbrina canosai, Sphyraena picudilla, Etropus intermedius, Symphurus trewavasae, Stephanolepis hispidus e Chilomecterus spinosus.
Paralonchurus brasiliensts ("Marıa Lurza ou "Rolo de Fumo") e Micropogon furnieri ("Corvina"), apresentam regime alimentar semelhante; o que coincide com observação de Vazzoler (1975) em Sciaenidae da plataforma continental, entre Torres e Chuí. Em 105 dos 112 exemplares de Paralonchurus brasiliensis, espécie que tem ocorrido com maior frequência e abundância, os poliquetos constituem o aliménto básico. Os poliquetos constituem cerca de $77,3 \%$ e $60,0 \%$ do volume total do alimento ingerido respectivamente por Paralonchurus brasiliensis e Micropogon furnieri (Fig. 2). 


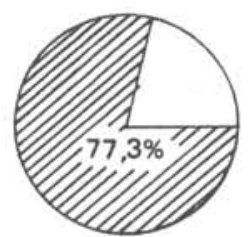

Paralonchurus brasiliensis

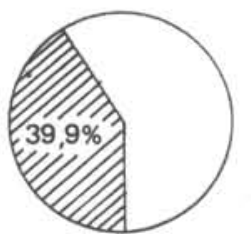

Orthopristis ruber

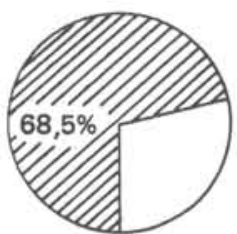

Micropogon furnieri

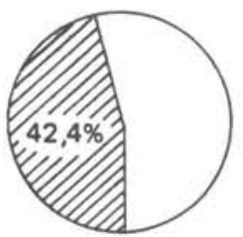

Menticirrhus americanus

Fig. 2 - Volume porcentual dos poliquetos encontrados no conteudo estomacal de Paralonchurus brasiliensis, Micropogon furnieri, Orthopristis ruber e Menticirrhus americanus.

$\mathrm{Na}$ alimentação da Orthopristis ruber ("Corcoroca") e Men. ticirrhus americanus ("Betara") os poliquetos são também de importância, com um volume superior a $40,0 \%$ (Fig. 2).

Através da análise sistemática dos anelídeos poliquetos encontrados nos estômagos pode-se identificar as seguintes espécies: Pholoë minuta, Sthenelais neoleanira, Sthenolepis oculata, Thalenessa levissii, Pseudeurythoë ambigua, Anaitides tamoya, Loandalia americana, Sigambra grubei, Langerhan sia cornuta, Glycera americana, Glycera longipinnis, Goniada maculata, Diopatra splendidissima, Nothria stigmatis, Onuphis eremita, Onuphis vexillaria, Eunice vi. tatta, Lysidice ninetta, Labrorostratus prolificus, Lumbrineris minima, Lumbrineris tetraura, Paradoneis armata, Apoprionospio dayi, Paraprionospio pinnata, Pherusa laevis, Pherusa parmata, Piromis arenosus, Travisia forbesii, Pectinaria laelia, Isolda pulchella, Loimia cf medusa, Thelepus cf setosus. Algumas destas espécies são comuns na região amostrada, enquanto que outras como Pholoe minuta, Sthenelais neoleanira, Anaitides tamoya, Nothria stigmatis, Paradoneis armata, Apoprionospio dayi e Travisia forbesii não foram referidas para a área. Caso estas espécies não sejam encontradas nas amostragens de fundo que estão sendo efetuadas simultaneamente aos arrastos, poderíamos supor que os organismos que utilizam esses anelídeos como alimento podem ter o hábito de se alimentar junto às rochas ou em regiões mais profundas.

Paralonchurus brasiliensis foi um dos peixes em que encontramos a maior variedade de gêneros e espécies; entre as 27 identificadas Pherusa parmata foi a mais frequente $(21,1 \%)$, seguida de Pherusa laevis $(8,1 \%)$ e Piromis arenosus $(10,6 \%)$ (Fig. 3). Estas três espécies pertencem à mesma família, Flabelligeridae, caracterizada pela presença de cerdas longas e fortes na região anterior e cujo corpo coberto por grãos de areia, justificaria terem sido encontradas em bom estado de conservação.

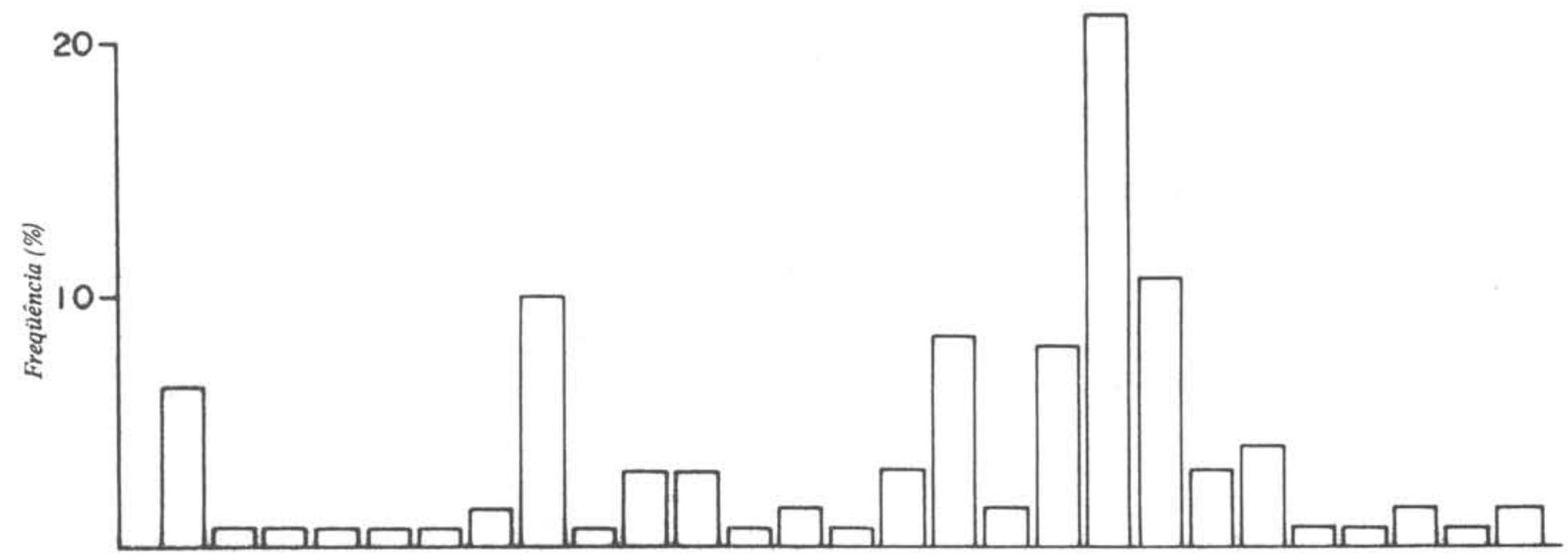

\begin{tabular}{|c|c|c|c|c|c|c|c|c|c|c|c|c|c|c|c|c|c|c|c|c|c|c|c|c|}
\hline 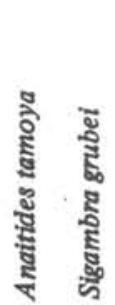 & 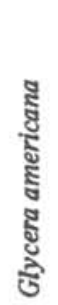 & 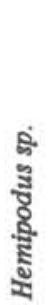 & 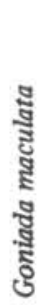 & 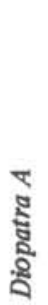 & 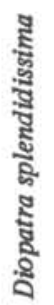 & $\begin{array}{l}\text { है } \\
\text { है } \\
\text { है } \\
\text { है } \\
\text { है } \\
\text { है }\end{array}$ & 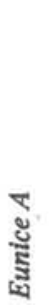 & 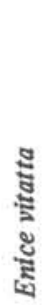 & 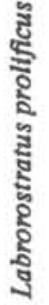 & 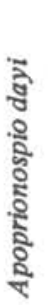 & 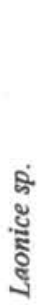 & 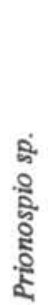 & 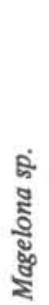 & के & $\begin{array}{l}\text { के } \\
\text { है } \\
\text { है }\end{array}$ & 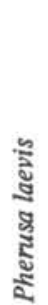 & 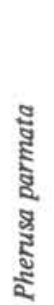 & 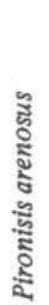 & 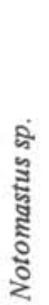 & 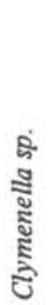 & $\begin{array}{l}\frac{9}{2} \\
\frac{1}{9} \\
\text { ș } \\
\text { ș } \\
\frac{0}{0}\end{array}$ & 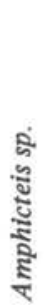 & 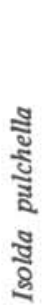 & 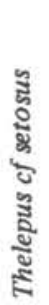 \\
\hline
\end{tabular}


Em Orthopristis ruber encontramos também um elevado número de espécies de poliquetos, cerca de 24 , dentre os quais Paraprionospio pinnata foi a mais frequente $(81,5 \%$ ) (Fig. 4). Pa. raprionospio pinnata esteve presente em todos estômagos analisados para um determinado arrasto, entretanto, o grande número de exemplares deste poliqueto pode ser atribuído ao fato de o cardume ter se alimentado em um único local, onde esta espécie deveria ser abundante.

Entre os linguados /Citharichthys cornutus, Cyclopsetta chittendeni. Etropus intermedius, Paraticntnys patagonicus, Scyacıum microrum. Scyacium papilossum, Symphurus diomedianus, Symphurus jenynsii, Symphurus plagusia, Symphurus trewavasae) a frequência de poliquetos foi mais alta em Etropus intermedius destacando-se Diopatra splendidissima (31.8\%) (Fig. 5 )

somente 8 espécies ae poliquetos foram wmuns a $U$. ruber e $P$. brasiliensis, o que talvez possa ser atribuído a hábitos alimenta. res diferentes. Orthopristis ruber alimenta-se com preferência em fundos rochosos e $P$. brasiliensis, provavelmente em fundos moles.

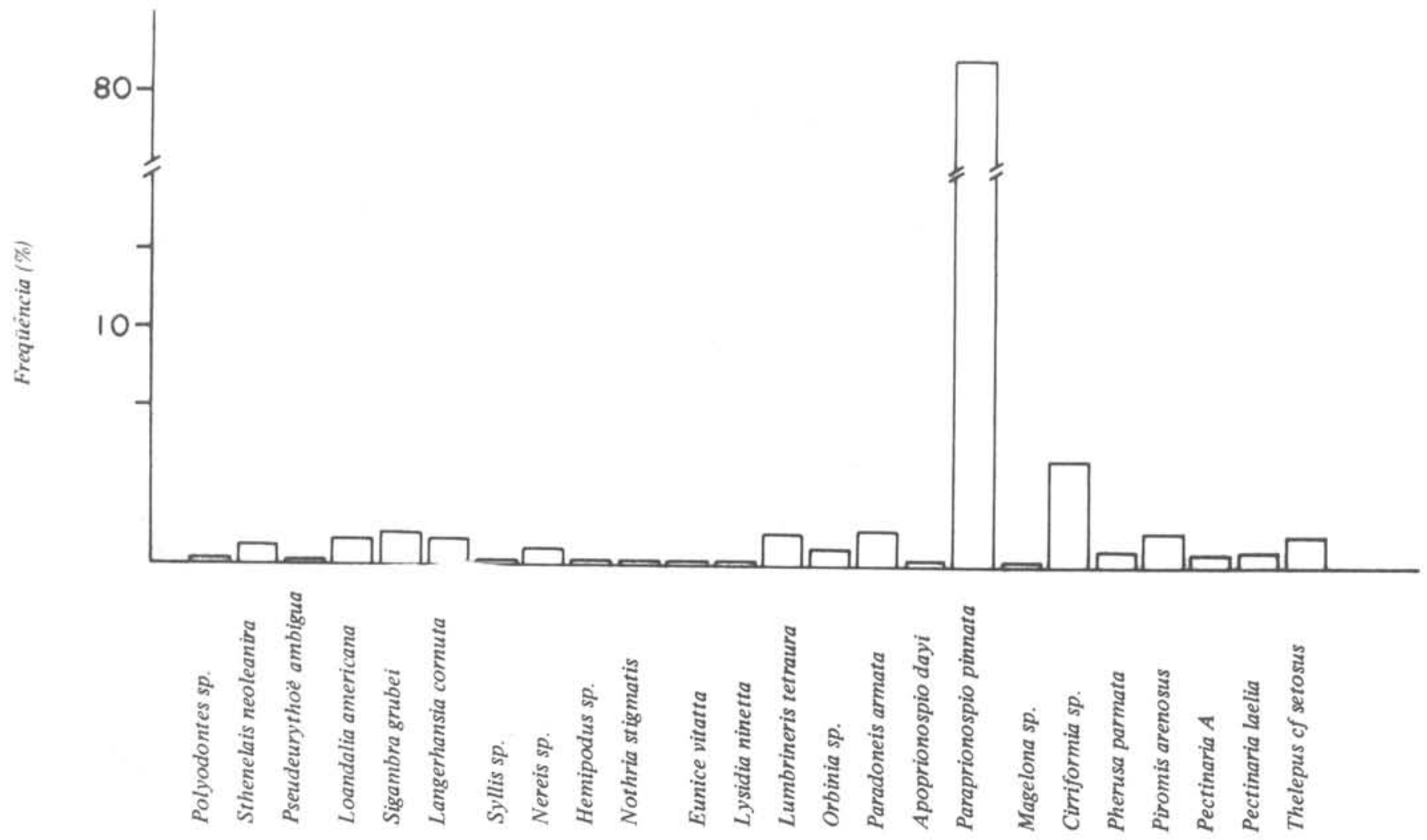

Fig. 4 - Freqüência de ocorrência dos gêneros e espécies de poliquetos encontrados no conteúdo estomacal de Orthopristis ruber.

A maioria dos peixes que se alimentou de poliquetos consumiu predominantemente Nothria stigmatis, Paraprionospio pinnata, Pherusa laevis, Piromis arenosus, Pectinaria laelia e Thelepus setosus; o que pode ser justificado pelo fato destas espécies serem comuns na região, ou mesmo pelos seus hábitos gregários facilitando sua captura pelo peixe. Thelepus setosus, por exemplo, pode formar extensos bancos em locals onde o fundo e composto por sedimentos areno-lodosos com fragmentos de conchas.

A ocorrência de Labrorostratus prolificus em alguns estômagos foi surpreendente pois, este poliqueto é um endoparasita e foi encontrado no celoma de um nereideo na regiâo de Ubatuba (Amaral, 1977); nos estômagos aqui analisados o hospedeiro pertencia à Família Eunicidae.

Randall (1967) havendo examinado o conteúdo estomacal de 212 espécies de peixes das Antilhas, concluiu que os poliquetos são uma das mais importantes fontes de alimento para peixes da região.
No Brasil, Kawakami \& Amaral (1976) mostraram que em algumas espécies'de linguado, os poliquetos constituem mais de $50 \%$ do conteúdo estomacal. Situação semelhante já havia sido constatada por Petersen (1915); ao fazer uma estimativa da produção bêntica do Kattegat, considerou que*os animais utéis (para alimentação de peixes e outros organismos de interesse econômico) alcançavam cerca de 1.000 .000 toneladas por ano, sendo a maioria constituída por poliquetos e pequenos bivalves.

Através dos resultados obtidos nesta primeira fase do projeto, podemos considerar que os poliquetos têm importante participação na alimentação de pelo menos 9 espécies de peixes demersais habitualmente pescados na região.

Ao fim do projeto, dispondo de maiores informações, que se espera sejam suficientes, sobre a distribuição dos poliquetos de fundo e sua importância como alimento, procurar-se-á correlacionar produtores e consumidores. 


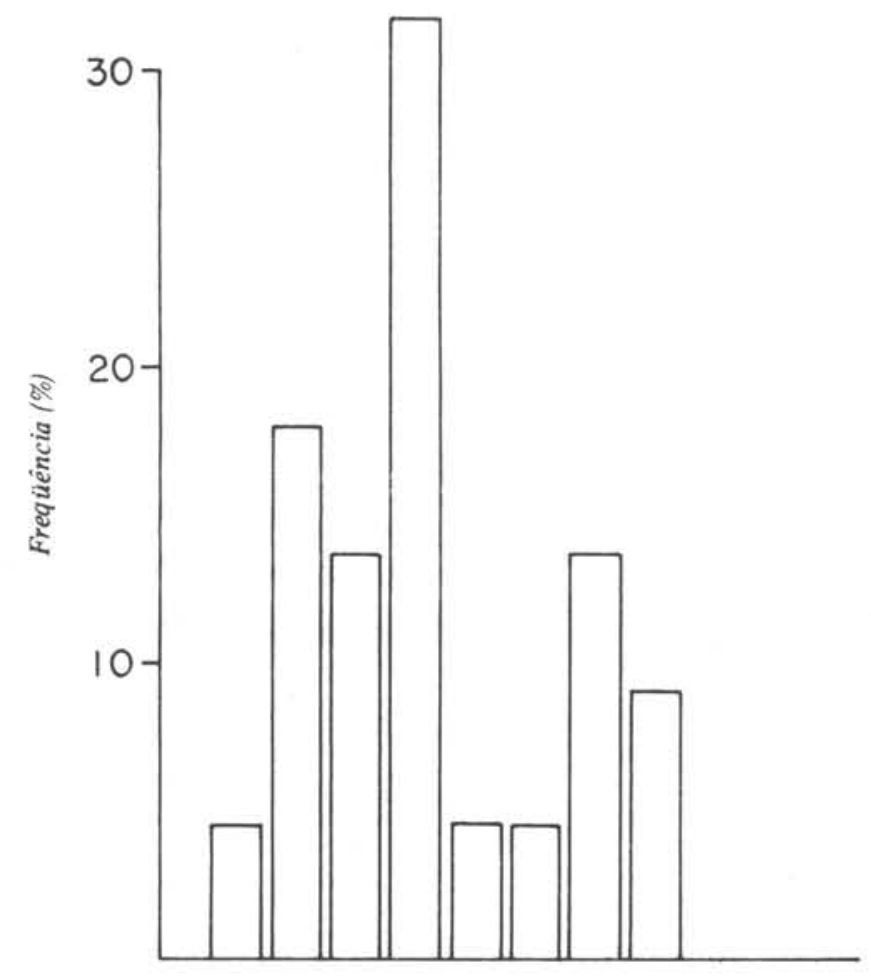

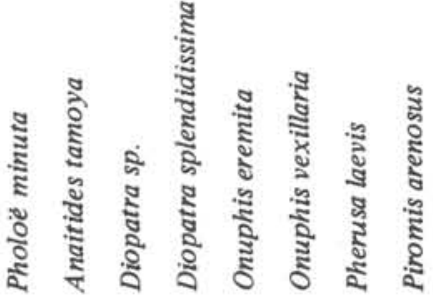

Fig. 5 - Freqüência de ocorrência dos generos e espécies de poliquetos encontrados no conteúdo estomacal de Etropus intermedius.

\section{Agradecimentos}

À Fundação de Amparo à Pesquisa do Estado de São Paulo, pelo apoio dado através da concessão de bolsa (Proc. Biológicas 77/1257). Ao Dr. Edmundo Ferraz Nonato pelo incentivo e dedicação constantes durante o desenvolvimento deste projeto. Ao Prof. Dr. Naércio A. Menezes, ao Lic. José Luis Figueiredo (Museu de Zoologia, Universidade de São Paulo), às colegas Elza Zaneti Prado e Suzana Anita Saccardo (Instituto Oceanográfico da Universidade de São Paulo) pelo auxílio prestado na identificação dos peixes. Ao Instituto Oceanográfico da Universidade de São Paulo, ao pessoal da Base Norte e tripulação do "Veliger II" pelas facilidades concedidas e auxílio nas coletas.

\section{Bibliografia}

AMARAL, A.C.Z. 1977. Um poliqueto endoparasita, Labrorostratus prolificus, sp. nov. em nereideo. Bolm Inst. oceanogr., S
Pauio, 26:285-292.

KAWAKAMI, E. \& AMARAL, A.C.Z. 1976. Importância dos anelídeos onliquetos regime alimentar de alguns pleuronectiformes. Ciênc. Cult., S. Paulo 28 (7):420 (resumo) 28a. Reunião Anual SBPC.

PERKINS, T.H. \& SAVAGE, T. 1975. A bibliography and check list of polychaetous annelids of Florida, the Gulf of Mexico and the Caribbean region. Florida Mar. Res. Publ., 14:1-62.

PETERSEN, C.J.G. 1915. On the animal communities of the sea botton in the Skagerak, the Christiania Fjord and the Danish waters. Rep. Danish Biol. Stat., 20:1-76.

RANDALL, J.E. 1967. Food habits of reef fishes of the West Indies. Stud. Trop. Oceanogr. (Miami), (5):665-847.

TAYLOR, J.L: \& SALOMAN, C.H. 1968. Rearing lug-worms for fish bait. Com. Fish Rev., 30(8-9):61-63.

VAZZOLER, G. 1975. Distribuição da fauna de peixes demersais e ecologia dos Sciaenidae da plataforma continental brasileira, entre as latitudes $29^{\circ} 21^{\prime} \mathrm{S}$ (Tôrres) e $33^{\circ} 41^{\prime} \mathrm{S}$ (Chuí). Bolm Inst. oceanogr. S. Paulo, 24:85-169. 